\title{
Distribución, abundancia, control y registros de casos de murciélagos vampiro, Desmodus rotundus (E. Geoffroy), infectados de rabia en ambientes pecuarios de Guerrero, México
}

Distribution, abundance, control, and rabies case records of infected vampire bats, Desmodus rotundus (E. Geoffroy), in livestock areas from Guerrero, Mexico

\author{
César Eduardo Romero-Barrera ${ }^{1}(\mathbb{D})$, Angel Neftali Osorio-Rodriguez ${ }^{2,3^{*}}(\mathbb{D})$, Alejandro Juárez-Agis ${ }^{4}(\mathbb{D}$
}

${ }^{1}$ Comité Estatal para el Fomento y Protección Pecuaria de Guerrero, S. C., Calle Pleasant Hill, núm. 17, Col. Burócratas 39090, Chilpancingo de los Bravo, Guerrero, México. ${ }^{2}$ Laboratorio Integral de Fauna Silvestre, Facultad de Ciencias Químico-Biológicas, Universidad Autónoma de Guerrero, Av. Lázaro Cárdenas s/n, Ciudad Universitaria Sur 39090, Chilpancingo de los Bravo, Guerrero, México. ${ }^{3}$ Instituto para el

Manejo y Conservación de la Biodiversidad, A. C., C. Durango, núm. 23, Col. José Vasconcelos, Chilpancingo de los Bravo, Guerrero, México. ${ }^{4}$ Ciencias Ambientales, Campus Llano Largo, Universidad Autónoma de Guerrero, Carretera Cayaco-Puerto Marquéz, Ejido Llano Largo, Parcela 56, 57 y 58, 3990, Acapulco, Guerrero, México.

*Autor para correspondencia: neftali.eptesicusfuscus@gmail.com

Fecha de recepción:

3 de mayo de 2020

Fecha de aceptación:

3 de agosto de 2020

Publicado en línea:

25 de marzo de 2021

Este es un artículo en acceso abierto que se distribuye de acuerdo a los términos de la licencia Creative Commons.

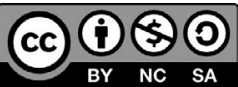

Reconocimiento-

NoComercial-

CompartirIgual 4.0

Internacional

\section{RESUMEN}

El murciélago vampiro, Desmodus rotundus (E. Geoffroy), es el principal vector de la rabia paralítica bovina, con amplia distribución en México. Ante la expansión de las actividades ganaderas en Guerrero, México, se desconoce el papel que podría desempeñar esta especie en la transmisión de la rabia paralítica. Por ello, con la finalidad de conocer la distribución y la abundancia del murciélago vampiro en los ambientes pecuarios, así como con la idea de analizar la importancia del control poblacional y registrar el número de murciélagos vampiro positivos al virus de la rabia en el estado de Guerrero, México, se capturaron 2,620 organismos, en 178 localidades, pertenecientes a 29 municipios, en el periodo 2013-2017. De los datos de abundancia, 30\% se clasificaron dentro de la categoría de "escaso". Se trataron con warfarina 2,482 (95\%) organismos; en 2017 se realizó el mayor número de tratamientos $(n=1,038)$. Se enviaron 136 especímenes a laboratorio, de los cuales cuatro (3\%) fueron positivos al virus rábico. Hasta la fecha, la campaña para prevenir la rabia paralítica bovina ha resultado una estrategia efectiva, considerando que no se han generado otras prácticas para mitigar la problemática que se genera entre esta especie hematófaga y las actividades pecuarias. Asimismo, es importante incluir campañas de concientización, con la finalidad de dar a conocer la importancia de la vacunación del ganado y la función ecológica de las demás especies de murciélagos.

\section{PALABRAS CLAVE}

Abundancia; rabia; tratamiento tópico; vampiricida; warfarina.

\section{ABSTRACT}

The vampire bat, Desmodus rotundus (E. Geoffroy), is the main vector of bovine paralytic rabies, widely distributed in Mexico. Given the expansion of livestock activities in Guerrero, Mexico, the role of this species in the transmission of paralytic rabies is unknown. In order to know the vampire bat distribution, abundance in livestock environments, to analyze the importance of population control, and to record the number of vampire bats with rabies in Guerrero, Mexico, 2,620 organisms were captured in 178 localities belonging to 29 municipalities, in the period 2013-2017. 30\% of the abundance data was classified into the sparse category. 2,482 (95\%) organisms were treated with warfarin, and in 2017 the largest number of treatments was performed ( $n=1,038)$. 136 samples were sent to the laboratory, four of which $(3 \%)$ tested positive for the rabies virus. The bovine paralytic rabies campaign has been an effective strategy so far, considering that no other strategies have been developed to mitigate the problems that this blood-sucking species cause for livestock 
activities. It is also important to include campaigns to raise awareness of the importance of livestock vaccination and the ecological role of other bat species.

\section{KEYWORDS}

Abundance; rabies, topical treatment, vampiricide, warfarine.

\section{INTRODUCCIÓN}

Las zoonosis afectan aproximadamente a $75 \%$ de la humanidad (Reyes-Novelo et al. 2011), como resultado de diversos factores ambientales y socioeconómicos que influyen en el comportamiento epidemiológico de las enfermedades (Soto-Estrada et al. 2016; Tapia-Conyer 2016); entre éstas, la rabia es una enfermedad reemergente que amenaza a México (Zarza et al. 2017; Paniagua 2018), un padecimiento letal ocasionado por un virus neurotrópico perteneciente a la familia Rhabdoviridae del género Lyssavirus (Paniagua 2018), el cual se caracteriza por la manera en que se manifiesta: rabia furiosa (encefalitis) y rabia muda (parálisis) (Badillo et al. 2009; Llamas y Orozco 2009).

La enfermedad se mantiene en dos ciclos: urbano y silvestre. En el primero, se encuentran ratas y ratones; sin embargo, si estos organismos estuvieran infectados del virus de la rabia difícilmente lo propagarían, pues presentan glándulas salivales rudimentarias; por lo tanto, es casi imposible que lo transmitan. Por otra parte, presentarían un cuadro clínico paralítico (Paniagua 2018) distinto al de los gatos (Felis catus L.) y perros (Canis lupus familiaris L.), los cuales pueden manifestar un cuadro clínico furioso o paralítico y sí pueden propagar el virus de la rabia (Llamas y Orozco 2009). En el segundo, se han reportado algunas especies de murciélagos insectívoros, frugívoros, nectarívoros, omnívoros, carnívoros y hematófagos (Escobar et al. 2015), además de mamíferos carnívoros como: coyotes (Canis latrans Say), zorros (Lycalopex griseus [Gray], Urocyon cinereoargenteus Schreber), zorrillos (Mephitis mephitis Schreber, Conepatus leuconotus Lichtenstein) y mapaches (Procyon lotor L.) (SS 2011).

El murciélago vampiro Desmodus rotundus (E. Geoffroy) es considerado el reservorio natural y transmisor del virus de la rabia en América Latina (Lee et al. 2012). En México, es el principal vector de la rabia paralítica bovina, resultado indirecto de su hábito alimenticio, debido a que, al morder y lamer la sangre, también inocula este virus (Anderson et al. 2012; Johnson et al. 2014; Zarza et al. 2017). Sin embargo, el porcentaje de rabia en estos organismos capturados ha sido bajo ( 10 a 14\%; Betancur et al. 2016; De Thoisy et al. 2016).

El estado de Guerrero ha sido considerado una zona endémica de rabia paralítica bovina, pues de 2017 a 2019 se reportaron 60 casos positivos en bovinos (SENASICA 2020); por otra parte, el murciélago vampiro es considerado como plaga por los ganaderos y campesinos, basados principalmente en su abundancia, determinada por el aumento de las actividades productivas como son la ganadería y agricultura. Esta relación (murciélago-ganado) representa una pérdida económica para los dueños del ganado, quienes toman medidas de mitigación no aptas (explosiones e incendios de refugios, así como envenenamientos), con lo cual afectan a otras especies de murciélagos no hematófagos - que cohabitan con el murciélago vampiro, e, incluso, perjudican refugios que no son habitados por esta especie hematófaga (Lord 1988; Anderson et al. 2012).

La Secretaría de Agricultura y Desarrollo Rural (SAGARPA) atendió este problema al consolidar el Comité Estatal para el Fomento y Protección Pecuaria de Guerrero S.C., un organismo auxiliar de salud animal. El personal técnico zoosanitario de este comité lleva a cabo campañas dirigidas al diagnóstico, la prevención y el control de la rabia transmitida a los bovinos y otras especies ganaderas, mediante la vacunación antirrábica del ganado susceptible y el control de las poblaciones de vampiros por tratamientos tópicos (SAGARPA 2011). Con la finalidad de generar el diagnóstico del murciélago vampiro (D. rotundus) en Guerrero, se procedió a determinar su distribución y abundancia en ambientes pecuarios, así como a evaluar la estrategia del control poblacional y a diagnosticar el número de individuos positivos al virus de la rabia. 


\section{Materiales y Métodos}

\section{Área de estudio}

La presente investigación se llevó a cabo en el estado de Guerrero, ubicado en el centro-sur de la República Mexicana, entre las coordenadas $16^{\circ} 18^{\prime}$ y $18^{\circ} 53^{\prime}$ de latitud norte y $98^{\circ} 03^{\prime}$ y $102^{\circ} 12^{\prime}$ de longitud oeste (Figura 1). El muestreo se realizó de enero de 2013 a diciembre de 2017, donde se visitaron 178 localidades (Anexo 1), las cuales fueron elegidas de manera aleatoria, tratando de cubrir la mayoría de las solicitudes generadas por campesinos y ganaderos del estado. Además, éstas se dirigieron estratégicamente a sitios donde meses o días previos habían reportado brotes de rabia paralítica bovina.

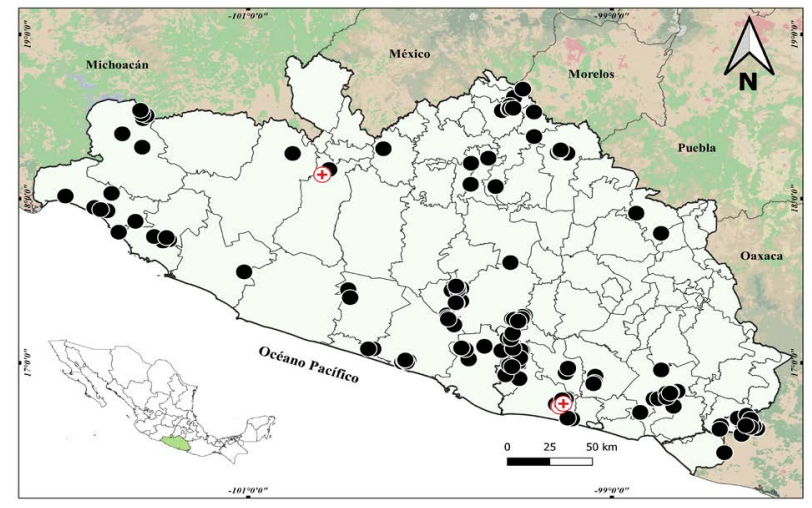

Figura 1. Ubicación del estado de Guerrero, México; los puntos negros representan los sitios de colecta del murciélago vampiro (D. rotundus) y los puntos rojos, resultados positivos al virus rábico de especímenes enviados a laboratorio.

\section{Captura de organismos}

En cada localidad, por noche, se seleccionó un corral de muestreo potencialmente usado por murciélagos vampiro, considerando que llegarían a alimentarse de la sangre del ganado. Al exterior de estos corrales se colocaron cuatro redes ornitológicas ( $12 \mathrm{~m}$ largo x $2.5 \mathrm{~m}$ alto), las cuales se desplegaron desde el nivel del suelo hasta aproximadamente $2.5 \mathrm{~m}$ de altura, con las bolsas de la red en dirección hacia el corral, para facilitar la captura. Las bolsas se abrieron después del crepúsculo y se cerraron hasta las 24:00 h. Fueron revisadas con base en la actividad y abundancia de los organismos. Se evitaron muestreos durante la fase de luna llena para reducir la fobia lunar.
Cada organismo capturado se identificó con la ayuda de la clave taxonómica para los murciélagos de México (Medellín et al. 2008); las especies de murciélagos no hematófagas fueron liberadas, y los murciélagos vampiro, depositados en una jaula especializada.

\section{Tratamiento tópico}

Para el control poblacional de estos organismos se utilizó un ungüento cuyo componente principal es la warfarina (anticoagulante), el cual se aplicó en el dorso y pecho de cada organismo capturado; posteriormente, fueron liberados, con la finalidad de propagar la sustancia entre el resto de su población, debido a sus hábitos de acicalamiento (Flores-Crespo 2003; Osorio-Rodriguez y Saldaña-Vázquez 2019). Cabe mencionar que el procedimiento se llevó a cabo respetando lo establecido por la Norma Oficial Mexicana NOM-067-ZOO-2007 (SAGARPA 2011).

\section{Diagnóstico rábico}

Fueron seleccionados individuos que por lo menos presentaran una de las siguientes características: comportamiento tranquilo, excesiva salivación y heridas recientes provocadas por otros murciélagos vampiro. Posteriormente, se apartaron en bolsas de tela para contenerlos, sacrificarlos y congelarlos. Por último, fueron enviados al Laboratorio Estatal de Salud Pública "Dr. Galo Soberón y Parra”, en Acapulco, Guerrero, y al Centro Nacional de Servicios de Diagnóstico en Salud Animal (CENASA), en el Estado de México, donde se les hizo el análisis de rabia por prueba de inmunofluorescencia directa (IFD); esta técnica se lleva a cabo por medio de un anticuerpo que se encuentra químicamente unido a una molécula fluorescente, la cual demuestra la presencia de la proteína del virus de la rabia.

\section{Análisis de datos.}

Para el procesamiento y análisis de la información, los datos fueron incorporados en una hoja de Microsoft Excel $^{\circledR}$ (versión 2016; para Windows) (Anexo 1). Se calculó el esfuerzo de muestreo de acuerdo con el método propuesto por Medellín (1993), a partir del 
Cuadro 1. Esfuerzo de muestreo, número de organismos capturados y tratados de murciélago vampiro (D. rotundus), a base de warfarina en Guerrero, México (2013-2017).

\begin{tabular}{cccccccc}
\hline Metros red * hora & 194,688 & 460,800 & 83,232 & 484,128 & 839,808 & Total \\
\hline Capturados & 317 & 543 & 199 & 486 & 1,075 & 2,620 \\
\hline Tratados & 294 & 520 & 175 & 455 & 1,038 & 2,482 \\
\hline
\end{tabular}

área neta (metros totales) y el número total de horas que las redes permanecieron abiertas; para este caso, el análisis se efectuó anualmente (Cuadro 1).

Para conocer el número de organismos de murciélagos vampiro capturados en cada localidad (abundancias), se ordenó la información de manera descendente, con la finalidad de generar un diagrama de burbujas con el software R-Studio ${ }^{\circledR}$ y la librería ggplot 2. Los datos se clasificaron en cuatro categorías: 1) abundante (30-100 organismos); 2) común (15-29 organismos); 3) poco abundante (5-14), y 4) escaso ( $<5$ organismos).

\section{RESUltados Y Discusión}

\section{Distribución y abundancia del murciélago vampiro D. rotundus}

En un periodo de cinco años se capturó un total de 2,620 organismos, provenientes de 178 localidades y 29 municipios (Cuadro 1; Anexo 1). El municipio con mayor número de localidades muestreadas fue Chilpancingo de los Bravo ( $\mathrm{n}=26)$; la localidad con mayor número de capturas fue Rancho el Cerro II (San Marcos) (localidad 147; Anexo 1), y el año con mayor número de capturas registradas fue 2017 ( $n=1,075$; Cuadro 1).

De acuerdo con los datos del Servicio de Información Agroalimentaria y Pesquera, en la última década, la ganadería en México ha tenido un aumento significativo; en Guerrero, se ha reportado un incremento de cabezas de ganado de $7.77 \%$ (SIAP 2020). Corroboramos lo antes mencionado debido a que se atendieron solicitudes en áreas que no eran consideradas ganaderas, así como con campesinos que anteriormente no ejercían esta actividad.

Por otra parte, las localidades de captura del murciélago vampiro están dispersas en 29 municipios del estado, lo que representa $36 \%$ del total; es posible que la ubicación geográfica del estado brinde las condiciones ambientales necesarias para la distribución del murciélago vampiro, tal como lo señalan Ceballos et al. (2006) y Zarza et al. (2017).

Diversos autores (Greenhall 1971; Delpietro et al. 1992; Quintana y Pacheco 2007; Johnson et al. 2014; Moya et al. 2015; Becker et al. 2018; Zortéa et al. 2018) mencionan que el murciélago vampiro es abundante en áreas donde existe presencia de ganado, debido a la disponibilidad y abundancia de éste, así como por su fácil detección. Aunado a ello, otras variables influyen en la presencia y abundancia de esta especie, como la disponibilidad de refugios naturales y artificiales (Greenhall et al. 1983; Flores-Crespo y Arellano-Sota 1991; Flores-Crespo 2003; Osorio-Rodriguez y Saldaña-Vázquez 2019).

Chilpancingo de los Bravo registró el mayor número de localidades muestreadas, como resultado de la cercanía de las oficinas del Comité Estatal para el Fomento y Protección Pecuaria de Guerrero; sin embargo, fue uno de los municipios que presentó un porcentaje menor de capturas $(5 \%)$, posiblemente debido al constante monitoreo y control de la zona.

Aunque este estudio no tiene como objetivo evaluar las incidencias y las características locales y de paisaje, las localidades más alejadas y con escasa presencia de actividades humanas fueron las que presentaron mayores afectaciones por mordeduras de murciélagos vampiro; asimismo, estas localidades presentaron mayores números de capturas, lo cual es similar a lo señalado por otros autores que estudiaron estos atributos en condiciones distintas a las del presente trabajo (Ávila-Flores et al. 2019; Lanzagorta-Valencia et al. 2020).

Para el número de capturas anuales, 41\% $(\mathrm{n}=1,075)$ fue registrado en 2017, debido a que fue el año en que se visitó el mayor número de localidades $(\mathrm{n}=54)$, lo cual influyó en el esfuerzo de muestreo.

La abundancia de murciélagos vampiro por localidad varió, desde 0 hasta 98 organismos (Anexo 1; Figura 2); por otra parte, $30 \%$ de estas localidades se 
clasifico dentro de la categoría de "escaso", seguida de $29 \%$ en la categoría "poco abundante" y por último las

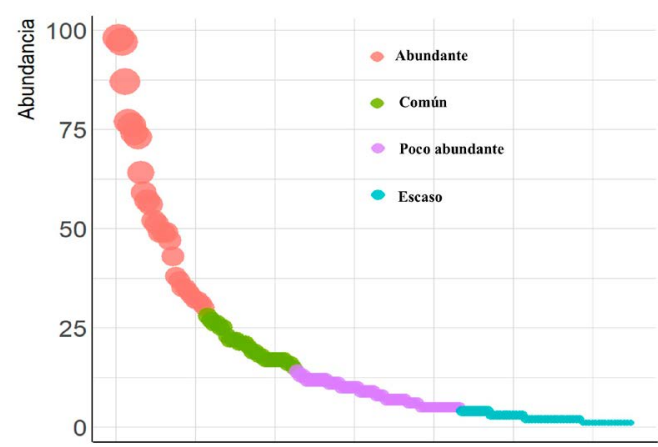

Figura 2. Número de organismos y categorías de abundancia de murciélagos vampiro (D. rotundus), colectados en ambientes pecuarios en Guerrero, México

categorías "común" y "abundante", con 15\% cada una (Figura 2). Como se observa, 59\% de las localidades estudiadas fueron clasificadas en las categorías de baja abundancia (escaso, poco abundante), y en $9 \%$ no se capturó organismo alguno; esto posiblemente se deba a que las localidades anteriormente habían tenido brotes de rabia paralítica bovina. Lo anterior concuerda con lo señalado por Delpietro y Russo (1996), quienes señalaron una disminución de $50 \%$ en las poblaciones del murciélago vampiro después de un brote rábico. Aunado a ello, la tasa baja de reproducción de esta especie hace que las poblaciones se recuperen de manera lenta (Johnson et al. 2014).

\section{Tratamiento tópico}

El total de murciélagos vampiro tratados fue de 2,482 (95\%; Cuadro 1); el mayor número de organismos tratados se registró en el municipio de Ometepec ( $\mathrm{n}=$ 387; Anexo 1). En cuanto a localidades, en el Rancho el Cerro II (San Marcos) y Rancho El Encanto (Ometepec), se trató el mayor número de organismos $(\mathrm{n}=97$; Anexo 1). El año con mayor número de tratamientos tópicos fue 2017 ( $\mathrm{n}=1,038$; Cuadro 1), valor similar a lo reportado en Nicaragua (Gonzalez y Mitchell 1976); asimismo estos autores reportan que, posterior al tratamiento tópico, el número de mordeduras disminuyó en $91.8 \%$. Aunque en el presente trabajo no se evaluó la reducción de mordeduras en el ganado, algunos ganaderos señalan que después del tratamiento tópico se redujeron las mordeduras. Lo anterior tiene sentido con lo mencionado por Flores-Crespo (2003), quien señala que bajo condiciones de laboratorio cada individuo tratado con ungüento de warfarina puede intoxicar a otros 20 organismos, además de que, en condiciones naturales, sucede igual e inclusive puede rebasar esta cifra. Sin embargo, estas cifras pueden variar, tal y como lo mencionan Osorio-Rodriguez y Saldaña-Vázquez (2019), quienes comentan que existen otras variables que influyen en la reducción de la población, por ejemplo, el cambio de la frecuencia de acicalamiento, influenciado por el microclima del refugio, el tamaño de percha y la fidelidad de grupo.

Johnson et al. (2014) sugieren que el control poblacional del murciélago vampiro mediante el tratamiento tópico a base de warfarina reduce temporalmente las poblaciones y la enfermedad, pero, a pesar de que esta actividad se ha efectuado por más de cuarenta años, los casos de rabia bovina han ido en aumento. Coincidimos, en parte, con esta aseveración; sin embargo, llevar a cabo esta actividad ha sido de gran apoyo para los ganaderos, debido a que existen áreas donde se ha reportado un alto número de agresiones al ganado, lo cual se ve reflejado en grandes pérdidas, como lo señala Anderson et al. (2012), y no sólo por los brotes rábicos, sino también por infecciones derivadas de las heridas (Schmidt y Badger 1979; Corrêa Scheffer et al. 2014).

Además, estas campañas han reducido hasta cierto punto la necesidad de que los ganaderos tomen medidas no aptas para el control del murciélago vampiro, las cuales afectan a otras especies de murciélagos no hematófagos (Greenhall 1971) que brindan distintos servicios ecosistémicos (control y supresión de insectos, polinización, dispersión de semillas).

\section{Diagnóstico rábico}

Se enviaron 136 especímenes a laboratorio, de los cuales, cuatro $(3 \%)$ fueron positivos a la prueba de IFD. Estos especímenes positivos fueron recolectados en los municipios de Ajuchitlán del Progreso y San Marcos (Anexo 1). Es claro que el número de muestras enviadas por localidad no es significativo, pues solamente representa $5 \%$ de los organismos 
capturados, además de que sólo se consideraron organismos que presentaron alguno de los criterios anteriormente mencionados, sin tener algún otro indicio visual de estar o no infectado.

Sin embargo, la prevalencia registrada (3\%) es similar a la reportada por SENASICA en enero-septiembre de 2017, donde analizaron 705 individuos de diferentes partes del país y de los cuales 13 fueron diagnosticados como casos positivos (2\%) (SENASICA 2017). Estos resultados y los documentados en el presente estudio soportan la hipótesis de que la prevalencia de rabia en $D$. rotundus es baja ( 10 a 14\%; Betancur et al. 2016; De Thoisy et al. 2016). Ante esto, es necesario incrementar el número de muestras para el análisis rábico para ofrecer un panorama más real de esta problemática a nivel estatal y nacional, e inclusive incluir organismos en varias categorías etarias (crías, jóvenes, adultos). Además, es indispensabe establecer vínculos con laboratorios que puedan llevar a cabo estos análisis, así como con instituciones educativas y de investigación para atender esta problemática.

\section{Conclusiones}

Se observó que el estado de Guerrero brinda las condiciones, topográficas y ambientales que permiten la distribución del murciélago vampiro (D. rotundus) en la mayor parte de la entidad. Por otra parte, los ambientes pecuarios del estado proveen de recursos alimenticios para la presencia y abundancia de esta especie.

El control poblacional por medio del tratamiento tópico resulta ser una estrategia efectiva, mientras se desarrollan mejores alternativas para mitigar esta problemática. Aunado a ello, se observó que el mismo virus rábico actúa como controlador de estas poblaciones, al disminuirlas.

El diagnostico nacional y estatal muestra una baja prevalencia del virus rábico en murciélagos vampiro; sin embargo, los casos de rabia paralítica bovina siguen aumentando.

Para la prevención de esta enfermedad se deben concentrar esfuerzos en la vacunación del ganado y especies domésticas que pueden ser vectores. Por otra parte, con la finalidad de mejorar el control poblacional de esta especie, deben incrementarse las campañas de concientización dirigidas a los ganaderos, para que vacunen al ganado, así como implementarse cursos y talleres sobre la diversidad e importancia de las especies de murciélagos no hematófagas.

\section{Agradecimientos}

Los autores agradecen al personal del Comité Estatal de Fomento y Protección Pecuaria del estado de Guerrero, por la operación del programa de vigilancia epidemiológica de la rabia bovina, en especial a Edelmiro Pérez Martínez, Rolando Cosme Santiago y Erik Gustavo Jaimes Catalán, por su gran aporte en el trabajo de campo. A Baltasar Cortés García, así como a Ricardo Villafuerte Muñoz, por su confianza y apoyo. Además, a Enrique Romero Carranza, por compartir su experiencia de 35 años de actividad en la campaña contra la rabia paralítica bovina. A José Alberto Almazán-Catalán, por sus comentarios. A los revisores, por sus comentarios para la mejora del escrito, así como al editor de esta revista. 


\section{LITERATURA CITADA}

Anderson A, Shwiff S, Gebhardt K, Ramírez AJ, Shwiff S, Kohler D, Lecuona L. 2012. Economic evaluation of vampire bat (Desmodus rotundus) rabies prevention in Mexico. Transboundary and Emerging Diseases 61: 140146. http://doi.org/10.1111/tbed.12007

Ávila-Flores R, Bolaina-Badal AL, Gallegos-Ruiz A, Sánchez-Gómez WS. 2019. Use of linear features by the common vampire bat (Desmodus rotundus) in a tropical cattle-ranching landscape. Therya 10: 229-234. https:// doi.org/10.12933/therya-19-890

Badillo R, Mantilla JC, Pradilla G. 2009. Encefalitis rábica humana por mordedura de murciélago en un área urbana de Colombia. Biomédica 29: 191-203.

Becker DJ, Czirják GÁ, Volokhov DV, Bentz AB, Carrera JE, Camus MS, Navara KJ, Chizhikov VE, Fenton MB, Simmons NB, Recuenco SE, Gilbert AT, Altizer S, Streicker DG. 2018. Livestock abundance predicts vampire bat demography, immune profiles and bacterial infection risk. Philosophical Transaction of the Royal Society B 373: 20170089. http://doi.org/10.1098/ rstb.2017.0089

Betancur C, Calderón A, Rodríguez VC. 2016. Presencia de virus rábico en murciélagos hematófagos en Colombia (Ciénaga de Oro y Sahagún, Córdoba). Biosalud 15: 1724. http://doi.org/10.17151/biosa.2016.15.1.3

Ceballos G, Blanco S, González C, Martínez E. [internet]. 2006. Desmodus rotundus (murciélago vampiro). Distribución potencial, escala 1:1000000. Instituto de Biología, Universidad Nacional Autónoma de México. México. [cited 2020 Marzo 30]. Disponible en: http:// www.conabio.gob.mx/informacion/gis/layouts/des_rotungw.png

Corrêa Scheffer K, Iamamoto K, Asano KM, Mori E, Garcia AIE, Achkar SM, Fahl WdeO. 2014. Murciélagos hematófagos como reservorios de la rabia. Revista Peruana de Medicina Experimental y Salud Pública 31: 302-309.

De Thoisy B, Bourhy H, Delaval M, Pontier D, Dacheux L, Darcissac E, Donato D, Guidez A, Larrous F, Lavenir R, Salmier A, Lacoste V, Lavergne A. 2016. Bioecological drivers of rabies virus circulation in a Neotropical bat community. PLOS Neglected Tropical Diseases 10: e0004378. http://doi.org/10.1371/journal.pntd.0004378

Delpietro HA, Russo RG. 1996. Aspectos ecológicos y epidemiológicos de la agresión del vampiro y de la rabia paralítica en la Argentina y análisis de las propuestas efectuadas para su control. Scientific and Technical Review of the Office International des Epizooties (Paris) 15: 971-984.

Delpietro HA, Marchevsky N, Simonetti E. 1992. Relative population densities and predation of the common vampire bat (Desmodus rotundus) in natural and cattle-raising areas in northeast Argentina. Preventive Veterinary Medicine 14: 13-20. http://doi. org/10.1016/0167-5877(92)90080-Y

Escobar LE, Peterson AT, Favi M, Yung V, Medina-Vogel G. 2015. Bat-borne rabies in Latin America. Revista do Instituto de Medicina Tropical Sao Paulo 57: 63-72. http://doi.org/10.1590/S0036-46652015000100009

Flores-Crespo R. 2003. Técnicas, substancias y estratégicas para el control de murciélagos vampiro. Organización Panamericana de la Salud/Organización Mundial de la Salud. Distrito Federal, México.

Flores-Crespo R, Arellano-Sota C. 1991. Biology and control of the vampire bat. In: Baer GM, editor. The Natural History of Rabies. Boca Raton, CRC Press. P. 461-476.

GonzalezR, MitchellGC.1976. Vampirebat control programs in Latin America. Proceedings of the 7th Vertebrate Pest Conference. Disponible en: https://digitalcommons.unl. edu/cgi/viewcontent.cgi? article=1021\&context=vpc7.

Greenhall AM. 1971. Lucha contra los murciélagos vampiros. Estudio y proyecto de programa para América Latina. Boletín de la Oficina Sanitaria Panamericana 71: 231-246.

Greenhall AM, Joermann G, Schmidt U. 1983. Desmodus rotundus. Mammalian Species 202: 1-6. https://doi. org/10.2307/3503895

Johnson N, Aréchiga-Ceballos N, Aguilar-Setien A. 2014. Vampire bat rabies: Ecology, epidemiology and control. Viruses 6: 1911-1928. http://doi.org/10.3390/v6051911

Lanzagorta-Valencia K, Fernández-Méndez JI, Medellín RA, Rodas-Martínez AZ, Ávila-Flores R. 2020. Landscape and cattle management attributes associated with the incidence of Desmodus rotundus attacks on cattle. Ecosistemas y Recursos Agropecuarios 7: 1-10. https:// doi.org/10.19136/era.a6n18.2164

Lee DN, Papeş M, Van Den Bussche RA. 2012. Present and potential future distribution of common vampire bats in the Americas and the associated risk to cattle. PLoS ONE 7: e42466. http://doi.org/10.1371/journal.pone.0042466

Llamas L, Orozco E. 2009. Rabia: infección viral del sistema nervioso central. Revista Mexicana de Neurociencia 10: 212-219. 
Lord RD. 1988. Control of vampire bats. In: Greenhall AM, Schmidt U, editors. Natural History of Vampire Bats. Florida, CRC Press. P. 2015-226.

Medellín RA. 1993. Estructura y diversidad de una comunidad de murciélagos en el trópico húmedo mexicano. En: Medellín RA, Ceballos G, editores. Avances en el estudio de los mamíferos de México. Publicaciones especies. Distrito Federal, Asociación Mexicana de Mastozoología, A. C. P. 333-354.

Medellín RA, Arita HT, Sánchez HO. 2008. Identificación de los murciélagos de México, clave de campo. Universidad Nacional Autónoma de México. Distrito Federal, México.

Moya MI, Pacheco LF, Aguirre LF. 2015. Relación de los ataques de Desmodus rotundus con el manejo del ganado caprino y algunas características del hábitat en la prepuna de Bolivia. Mastozoología Neotropical 22: 73-84.

Osorio-Rodriguez AN, Saldaña-Vázquez RA. 2019. Control del murciélago vampiro (Desmodus rotundus) en México: ¿Qué tan efectiva es para controlar la rabia bovina? En: Ornelas-García CP, Álvarez F, Wegier A, editores. Antropización: primer análisis integral. Ciudad de México, Instituto de Biología-Universidad Nacional Autónoma de México/Consejo Nacional de Ciencia y Tecnología. P. 409-418.

Paniagua HR. 2018. Rabia. En: Aburto-López AI, Galicia FJ, editores. Principales problemas de salud pública en México. Ciudad de México, Universidad Nacional Autónoma de México. P. 393-418.

Quintana H, Pacheco V. 2007. Identificación y distribución de los murciélagos vampiros del Perú. Revista Peruana de Medicina Experimental y Salud Publica 24: 81-88. https://doi.org/10.17843/rpmesp.2007.241.1084

Reyes-Novelo E, Ruíz-Piña $\mathrm{H}$, Escobedo-Ortegón J, Rodríguez-Vivas I, Bolio-González M, PolancoRodríguez Á, Manrique-Saide P. 2011. Situación actual y perspectivas para el estudio de las enfermedades zoonóticas emergentes, reemergentes y olvidadas en la Península de Yucatán, México. Tropical and Subtropical Agroecosystems 14: 35-54.

[SAGARPA] Secretaría de Agricultura, Ganadería, Desarrollo Rural, Pesca y Alimentación. [internet]. 2011. Norma Oficial Mexicana NOM-067-ZOO-2007, Campaña nacional para la prevención y control de la rabia en bovinos y especies ganaderas. Diario Oficial de la Federación 2011. [cited 2020 marzo 30]. Disponible en: http://www.dof.gob.mx/normasOficiales/4385/sagarpa/ sagarpa.htm.
Schmidt KM, Badger DD. 1979. Some social and economic aspects in controlling vampire bats. Proceedings of the Oklahoma Academy of Science 59: 112-114.

[SENASICA] Servicio Nacional de Sanidad Inocuidad y Calidad Agroalimentaria. [internet]. 2017. Campaña Nacional para la prevención y control de la rabia en bovinos y especies ganaderas. [cited 2019 enero 1]. Disponible en: https://www.gob.mx/senasica/ documentos/indicadores-de-la-campana-nacional-para-la-prevencion-y-control-de-la-rabia-en-bovinos-y-especies-ganaderas-2017.

[SENASICA] Servicio Nacional de Sanidad Inocuidad y Calidad Agroalimentaria. [internet]. 2020. Rabia paralítica. [cited 2020 julio 25]. Disponible en: https:// www.gob.mx/senasica/acciones-y-programas/campana-nacional-para-la-prevencion-y-control-de-la-rabia-en-bovinos-y-especies-ganaderas.

[SIAP] Servicio de Información Agroalimentaria y Pesquera. [internet]. 2020. Población ganadera 20102019 cabezas. [cited 2020 julio 25]. Disponible en: https://www.gob.mx/cms/uploads/attachment/ file/564340/Inventario_2019_bovinos.pdf

Soto-Estrada G, Moreno-Altamirano L, Pahua D. 2016. Panorama epidemiológico de México, principales causas de morbilidad y mortalidad. Revista de la Facultad de Medicina 59: 8-22.

[SS] Secretaría de Salud. [internet]. 2011. Diario Oficial de la Federación, de la Norma Oficial Mexicana NOM011-SSA2-2011. Para la prevención y control de la rabia humana y en los perros y gatos. [cited 2020 marzo 30]. Disponible en: http://www.cenaprece.salud. gob.mx/programas/interior/zoonosis/descargas/pdf/ NOM-011-SSA2-2011.pdf

Tapia-Conyer R. 2016. Una visión crítica sobre la salud pública en México. Gaceta Médica México 152: 278-284.

Zarza H, Martínez-Meyer E, Suzán G, Ceballos G. 2017. Geographic distribution of Desmodus rotundus in Mexico under current and future climate change scenarios: Implications for bovine paralytic rabies infection. Veterinaria México OA 4(3): 1-16. http://doi. org/10.21753/vmoa.4.3.390

Zortéa M, Silva DA, Calaça AM. 2018. Susceptibility of targets to the vampire bat Desmodus rotundus are proportional to their abundance in Atlantic Forest fragments? Iheringia. Série Zoologia 108: e2018037. http:// doi.org/10.1590/1678-4766e2018037 
Anexo 1. Localidades de captura, tratamientos realizados y casos rábicos positivos asociados al murciélago vampiro, Desmodus rotundus, en Guerrero, México.

\begin{tabular}{|c|c|c|c|}
\hline Coordenadas geográficas & Capturados & Tratados & Año de muestreo \\
\hline \multicolumn{4}{|l|}{ Acapulco de Juárez } \\
\hline $17^{\circ} 6^{\prime} 34.81^{\prime \prime N}, 99^{\circ} 33^{\prime} 3.64^{\prime \prime O}, 324$ msnm & 5 & 5 & 2014 \\
\hline $17^{\circ} 6{ }^{\prime} 2.92^{\prime \prime} \mathrm{N}, 99^{\circ} 42^{\prime} 3.14^{\prime \prime O}, 579 \mathrm{msnm}$ & 17 & 17 & 2014 \\
\hline $17^{\circ} 5^{\prime} 26.62^{\prime \prime} \mathrm{N}, 99^{\circ} 49^{\prime} 37.83^{\prime \prime} \mathrm{O}, 724 \mathrm{msnm}$ & 5 & 5 & 2017 \\
\hline $17^{\circ} 4^{\prime} 51.47^{\prime \prime} \mathrm{N}, 99^{\circ} 48^{\prime} 17.76^{\prime \prime} \mathrm{O}, 724 \mathrm{msnm}$ & $12^{(1)}$ & 11 & 2017 \\
\hline $17^{\circ} 4^{\prime} 27.71^{\prime \prime N}, 99^{\circ} 36^{\prime} 22.16^{\prime \prime O}, 274$ msnm & $12^{(3)}$ & 9 & 2014 \\
\hline $17^{\circ} 2^{\prime} 32.64^{\prime \prime} \mathrm{N}, 9^{\circ} 33^{\prime} 53.68^{\prime \prime} \mathrm{O}, 181 \mathrm{msnm}$ & $28^{(2)}$ & 26 & 2014 \\
\hline $17^{\circ} 1^{\prime} 30.03^{\prime \prime} \mathrm{N}, 99^{\circ} 47^{\prime} 5.83^{\prime \prime} \mathrm{O}, 370 \mathrm{msnm}$ & $51^{(1)}$ & 50 & 2017 \\
\hline $17^{\circ} 1^{\prime} 23.81^{\prime \prime} \mathrm{N}, 99^{\circ} 47^{\prime} 13.60^{\prime \prime} \mathrm{O}, 363 \mathrm{msnm}$ & 49 & 49 & 2017 \\
\hline $16^{\circ} 55^{\prime} 34.76^{\prime \prime N}, 99^{\circ} 35^{\prime} 4.70^{\prime \prime O}, 230 \mathrm{msnm}$ & 52 & 52 & 2017 \\
\hline \multicolumn{4}{|l|}{ Ajuchitlán del Progreso } \\
\hline $18^{\circ} 10^{\prime} 37.65 " \mathrm{~N}, 100^{\circ} 33^{\prime} 18.11^{\prime \prime O}, 295$ msnm & 0 & 0 & 2014 \\
\hline $18^{\circ} 10^{\prime} 25.45^{\prime \prime} \mathrm{N}, 100^{\circ} 33^{\prime} 15.47^{\prime \prime} \mathrm{O}, 299 \mathrm{msnm}$ & 1 & 1 & 2014 \\
\hline $18^{\circ} 8^{\prime} 44.68^{\prime \prime N}, 100^{\circ} 35^{\prime} 34.30^{\prime \prime O}, 384 \mathrm{msnm}^{*}$ & $43^{(2)^{*}}$ & 41 & 2015 \\
\hline \multicolumn{4}{|l|}{ Arcelia } \\
\hline $18^{\circ} 18^{\prime} 14.33^{\prime \prime N}, 100^{\circ} 15^{\prime} 22.46 " \mathrm{O}, 447$ msnm & 1 & 1 & 2013 \\
\hline \multicolumn{4}{|l|}{ Atoyac de Álvarez } \\
\hline $17^{\circ} 26^{\prime} 43.13^{\prime \prime} \mathrm{N}, 100^{\circ} 26^{\prime} 58.74^{\prime \prime O}, 813 \mathrm{msnm}$ & $17^{(2)}$ & 15 & 2015 \\
\hline $17^{\circ} 23^{\prime} 49.96 " \mathrm{~N}, 100^{\circ} 26^{\prime} 21.27^{\prime \prime O}, 650 \mathrm{msnm}$ & 1 & 1 & 2017 \\
\hline $17^{\circ} 5^{\prime} 6.38^{\prime \prime} \mathrm{N}, 100^{\circ} 20^{\prime} 22.82^{\prime \prime} \mathrm{O}, 18 \mathrm{msnm}$ & $3^{(1)}$ & 2 & 2017 \\
\hline $17^{\circ} 5^{\prime} 6.07^{\prime \prime} \mathrm{N}, 100^{\circ} 18^{\prime} 41.544^{\prime \prime O}, 31 \mathrm{msnm}$ & $11^{(1)}$ & 10 & 2017 \\
\hline \multicolumn{4}{|l|}{ Ayutla de los Libres } \\
\hline $16^{\circ} 55^{\prime} 7.56^{\prime \prime} \mathrm{N}, 99^{\circ} 5^{\prime} 28.29^{\prime \prime} \mathrm{O}, 359 \mathrm{msnm}$ & $26^{(2)}$ & 24 & 2013 \\
\hline $16^{\circ} 52^{\prime} 50.05^{\prime \prime N}, 99^{\circ} 5^{\prime} 41.78^{\prime \prime O}, 222 \mathrm{msnm}$ & 4 & 4 & 2016 \\
\hline $16^{\circ} 52^{\prime 22.26 " N, ~ 99 \circ 6 ' 3.06 " O, ~} 192$ msnm & $7^{(2)}$ & 5 & 2016 \\
\hline $16^{\circ} 52^{\prime} 12.64^{\prime \prime N}, 99^{\circ} 5^{\prime} 44.26^{\prime \prime O}, 214$ msnm & 5 & 5 & 2013 \\
\hline \multicolumn{4}{|l|}{ Azoyú } \\
\hline $16^{\circ} 44^{\prime} 7.51 " \mathrm{~N}, 98^{\circ} 39^{\prime} 37.91 " \mathrm{O}, 444 \mathrm{msnm}$ & $5^{(1)}$ & 4 & 2016 \\
\hline \multicolumn{4}{|l|}{ Buenavista de Cuéllar } \\
\hline $18^{\circ} 31^{\prime} 37.10^{\prime \prime N}, 9^{\circ} 25^{\prime} 40.57^{\prime \prime O}, 1117 \mathrm{msnm}$ & 1 & 1 & 2015 \\
\hline $18^{\circ} 22^{\prime} 40.41^{\prime \prime N}, 9^{\circ} 25^{\prime} 39.47^{\prime \prime O}, 1073$ msnm & $37^{(1)}$ & 36 & 2013 \\
\hline \multicolumn{4}{|l|}{ Chilpancingo de los Bravo } \\
\hline $17^{\circ} 28^{\prime} 2.75^{\prime \prime} \mathrm{N}, 99^{\circ} 51^{\prime} 23.47^{\prime \prime O}, 861 \mathrm{msnm}$ & $2^{(1)}$ & 1 & 2017 \\
\hline $17^{\circ} 28^{\prime} 1.09^{\prime \prime N}, 99^{\circ} 51 ' 22.64^{\prime \prime O}, 825$ msnm & $6^{(1)}$ & 5 & 2016 \\
\hline $17^{\circ} 27^{\prime} 30.82^{\prime \prime} \mathrm{N}, 99^{\circ} 50^{\prime} 57.44^{\prime \prime} \mathrm{O}, 762 \mathrm{msnm}$ & $1^{(1)}$ & 0 & 2017 \\
\hline $17^{\circ} 27^{\prime} 27.59^{\prime \prime N}, 99^{\circ} 52^{\prime} 32.66^{\prime \prime O}, 829 \mathrm{msnm}$ & 4 & 4 & 2014 \\
\hline $17^{\circ} 27^{\prime} 11.20^{\prime \prime N}, 9^{\circ} 49^{\prime} 44.83^{\prime \prime} \mathrm{O}, 753 \mathrm{msnm}$ & $17^{(1)}$ & 16 & 2017 \\
\hline $17^{\circ} 26^{\prime} 31.06^{\prime \prime N}, 99^{\circ} 52^{\prime} 49.73 " \mathrm{O}, 914 \mathrm{msnm}$ & 3 & 3 & 2014 \\
\hline $17^{\circ} 26^{\prime} 30.91^{\prime \prime N}, 99^{\circ} 52^{\prime} 49.57^{\prime \prime O}, 949$ msnm & 1 & 1 & 2016 \\
\hline
\end{tabular}


$17^{\circ} 26^{\prime} 30.80^{\prime \prime} \mathrm{N}, 9^{\circ} 50^{\prime} 54.51 " \mathrm{O}, 748 \mathrm{msnm}$ $17^{\circ} 26^{\prime} 30.18^{\prime \prime} \mathrm{N}, 9^{\circ} 50^{\prime} 50.76^{\prime \prime} \mathrm{O}, 774 \mathrm{msnm}$ $17^{\circ} 22^{\prime} 32.32 " \mathrm{~N}, 9^{\circ} 49^{\prime} 45.67^{\prime \prime} \mathrm{O}, 900 \mathrm{msnm}$ $17^{\circ} 21^{\prime} 56.83^{\prime \prime} \mathrm{N}, 9^{\circ} 51^{\prime 2} 28.09^{\prime \prime} \mathrm{O}, 1008 \mathrm{msnm}$ $17^{\circ} 21^{\prime} 45.60^{\prime \prime} \mathrm{N}, 99^{\circ} 51^{\prime} 17.91 " \mathrm{O}, 1024 \mathrm{msnm}$ $17^{\circ} 17^{\prime} 32.54^{\prime \prime N}, 9^{\circ} 54 ' 25.71 " \mathrm{O}, 954 \mathrm{msnm}$ $17^{\circ} 17^{\prime} 17.45^{\prime \prime} \mathrm{N}, 9^{\circ} 28^{\prime} 44.766^{\prime \prime} \mathrm{O}, 730 \mathrm{msnm}$ $17^{\circ} 17^{\prime} 12.07^{\prime \prime} \mathrm{N}, 9^{\circ} 54^{\prime} 42.62^{\prime \prime} \mathrm{O}, 887 \mathrm{msnm}$ $17^{\circ} 17^{\prime} 11.29^{\prime \prime N}, 9^{\circ} 28^{\prime} 56.93^{\prime \prime O}, 725$ msnm $17^{\circ} 17^{\prime} 0.22^{\prime \prime} \mathrm{N}, 9^{\circ} 29^{\prime} 28.54^{\prime \prime} \mathrm{O}, 730 \mathrm{msnm}$ $17^{\circ} 16^{\prime} 59.78^{\prime \prime} \mathrm{N}, 9^{\circ} 29^{\prime} 31.59^{\prime \prime} \mathrm{O}, 728 \mathrm{msnm}$ $17^{\circ} 16^{\prime} 54.26^{\prime \prime} \mathrm{N}, 9^{\circ} 29^{\prime} 44.88^{\prime \prime} \mathrm{O}, 728 \mathrm{msnm}$ $17^{\circ} 16^{\prime} 11.91 " \mathrm{~N}, 9^{\circ} 29^{\prime} 44.88^{\prime \prime O}, 709$ msnm $17^{\circ} 16^{\prime} 6.74{ }^{\prime \prime N}, 99^{\circ} 31^{\prime} 45.66^{\prime \prime O}, 679$ msnm $17^{\circ} 16^{\prime} 1.09^{\prime \prime} \mathrm{N}, 99^{\circ} 32^{\prime} 52.55^{\prime \prime} \mathrm{O}, 679 \mathrm{msnm}$ $17^{\circ} 15^{\prime} 55.73^{\prime \prime} \mathrm{N}, 99^{\circ} 32^{\prime} 15.04 " \mathrm{O}, 691 \mathrm{msnm}$ $17^{\circ} 15^{\prime} 51.77^{\prime \prime N}, 99^{\circ} 32^{\prime} 2.25^{\prime \prime O}, 703$, msnm $17^{\circ} 15^{\prime} 42.23^{\prime \prime N}, 9^{\circ} 31^{\prime} 42.67^{\prime \prime O}, 729 \mathrm{msnm}$ $17^{\circ} 15^{\prime} 14.11^{\prime \prime N}, 99^{\circ} 30^{\prime} 53.06^{\prime \prime O}, 712$ msnm

\section{Coahuayutla de José María Izazaga}

18³2'16.65"N, 101³5'33.81"O, 712 msnm $18^{\circ} 31^{\prime} 38.72^{\prime \prime N}, 101^{\circ} 35^{\prime} 26.38^{\prime \prime O}, 716$ msnm $18^{\circ} 31^{\prime} 21.15^{\prime \prime N}, 101^{\circ} 35^{\prime} 18.99 " \mathrm{O}, 717 \mathrm{msnm}$ $18^{\circ} 30^{\prime} 50.74 " \mathrm{~N}, 101^{\circ} 35^{\prime} 0.39^{\prime \prime} \mathrm{O}, 725 \mathrm{msnm}$ $18^{\circ} 30^{\prime} 21.31^{\prime \prime N}, 101^{\circ} 33^{\prime} 35.50 " \mathrm{O}, 742 \mathrm{msnm}$ $18^{\circ} 29^{\prime} 13.14^{\prime \prime} \mathrm{N}, 101^{\circ} 34^{\prime} 41.08^{\prime \prime} \mathrm{O}, 736 \mathrm{msnm}$ $18^{\circ} 23^{\prime} 41.46^{\prime \prime} \mathrm{N}, 101^{\circ} 41^{\prime} 32.677^{\prime \prime} \mathrm{O}, 694 \mathrm{msnm}$ $18^{\circ} 18^{\prime} 47.13^{\prime \prime N}, 101^{\circ} 34^{\prime} 58.34$ "O, 789 msnm

\section{Cocula}

$18^{\circ} 14^{\prime} 46.00^{\prime \prime} \mathrm{N}, 99^{\circ} 40^{\prime} 45.08^{\prime \prime} \mathrm{O}, 638 \mathrm{msnm}$ $18^{\circ} 5^{\prime} 13.68^{\prime \prime} \mathrm{N}, 9^{\circ} 46^{\prime} 35.10^{\prime \prime O}, 724 \mathrm{msnm}$ $18^{\circ} 5^{\prime} 8.30^{\prime \prime} \mathrm{N}, 99^{\circ} 46^{\prime} 39.43^{\prime \prime O}, 743 \mathrm{msnm}$

\section{Coyuca de Benítez}

17²16'30.00"N, 9954'17.03"O, 851 msnm $17^{\circ} 15^{\prime} 58.21^{\prime \prime N}, 99^{\circ} 54^{\prime} 0.12^{\prime \prime O}, 819$ msnm $17^{\circ} 14^{\prime} 28.18^{\prime \prime} \mathrm{N}, 99^{\circ} 52^{\prime} 19.42^{\prime \prime} \mathrm{O}, 780 \mathrm{msnm}$ $17^{\circ} 14^{\prime} 20.85^{\prime \prime} \mathrm{N}, 9^{\circ} 52^{\prime} 17.01^{\prime \prime} \mathrm{O}, 778 \mathrm{msnm}$ $17^{\circ} 14^{\prime} 3.73^{\prime \prime N}, 9^{\circ} 51^{\prime} 42.52^{\prime \prime O}, 804$ msnm $17^{\circ} 13^{\prime} 57.22^{\prime \prime} \mathrm{N}, 99^{\circ} 52^{\prime} 3.99^{\prime \prime} \mathrm{O}, 787 \mathrm{msnm}$ $17^{\circ} 13^{\prime} 51.41^{\prime \prime N}, 99^{\circ} 52^{\prime} 3.34^{\prime \prime O}, 799$ msnm $17^{\circ} 13^{\prime} 49.93^{\prime \prime} \mathrm{N}, 99^{\circ} 52^{\prime} 12.10^{\prime \prime O}, 803 \mathrm{msnm}$ $17^{\circ} 1$ '2.30"N, $100^{\circ} 88^{\prime} 8.53$ "O, $154 \mathrm{msnm}$ $17^{\circ} 0{ }^{\prime} 52.33^{\prime \prime} \mathrm{N}, 100^{\circ} 8^{\prime} 26.26^{\prime \prime O}, 209 \mathrm{msnm}$ $17^{\circ} 0^{\prime} 44.28^{\prime \prime} \mathrm{N}, 100^{\circ} 7^{\prime} 9.55^{\prime \prime} \mathrm{O}, 31 \mathrm{msnm}$ $17^{\circ} 0{ }^{\prime} 15.56^{\prime \prime} \mathrm{N}, 100^{\circ} 7^{\prime} 40.41^{\prime \prime} \mathrm{O}, 12 \mathrm{msnm}$ $17^{\circ} 0^{\prime} 15.48^{\prime \prime} \mathrm{N}, 100^{\circ} 7^{\prime} 40.26^{\prime \prime O}, 12 \mathrm{msnm}$ $17^{\circ} 0^{\prime} 15.19^{\prime \prime} \mathrm{N}, 100^{\circ} 9^{\prime} 9.58^{\prime \prime} \mathrm{O}, 22 \mathrm{msnm}$

\section{Coyuca de Catalán}

$18^{\circ} 16^{\prime} 30.10^{\prime \prime} \mathrm{N}, 100^{\circ} 45^{\prime} 19.68^{\prime \prime} \mathrm{O}, 318 \mathrm{msnm}$

\section{Cuajinicuilapa}

$\begin{array}{lll}7^{(1)} & 6 & 2017 \\ 2^{(1)} & 2 & 2017 \\ 3^{(1)} & 2 & 2017 \\ 5^{(1)} & 0 & 2017 \\ 19^{(3)} & 4 & 2017 \\ 15^{(1)} & 16 & 2015 \\ 1^{(1)} & 14 & 2016 \\ 3^{(1)} & 0 & 2015 \\ 1^{(1)} & 3 & 2016 \\ 2^{(1)} & 1 & 2016 \\ 17^{(1)} & 1 & 2016 \\ 1^{(1)} & 17 & 2014 \\ 9^{(1)} & 0 & 2017 \\ 3^{(1)} & 16 & 2017 \\ 2^{(1)} & 9 & 2017 \\ 2 & 2 & 2017 \\ 1^{(1)} & 2 & 2017 \\ & 2 & 2016 \\ & 1 & 2017\end{array}$

$\begin{array}{lll}9^{(1)} & 8 & 2016 \\ 0 & 0 & 2016 \\ 0 & 0 & 2016 \\ 6^{(1)} & 5 & 2016 \\ 10^{(1)} & 9 & 2016 \\ 6 & 6 & 2016 \\ 0 & 0 & 2013 \\ 0 & 0 & 2016\end{array}$

2016

2017

2017

2015

2015

2014

2014

2014

2014

2014

2015

2014

2014

2013

2014

2013

2013

$2^{(1)}$

$16^{(3)}$

13

2016

7

7

$16^{\circ} 27^{\prime} 9.95^{\prime \prime} \mathrm{N}, 98^{\circ} 22^{\prime} 50.17^{\prime \prime} \mathrm{O}, 77 \mathrm{msnm}$
2016 


\section{Cuautepec}

$16^{\circ} 41^{\prime} 52.48^{\prime \prime} \mathrm{N}, 98^{\circ} 50^{\prime} 37.28^{\prime \prime} \mathrm{O}, 152 \mathrm{msnm}$

\section{Cuetzala del Progreso}

$18^{\circ} 12^{\prime} 55.40^{\prime \prime} \mathrm{N}, 9^{\circ} 46^{\prime} 28.06^{\prime \prime} \mathrm{O}, 1717 \mathrm{msnm}$

\section{Eduardo Neri}

\section{Huitzuco de los Figueroa}

$18^{\circ} 17^{\prime} 47.18^{\prime \prime} \mathrm{N}, 99^{\circ} 16^{\prime} 53.29^{\prime \prime O}, 996$ msnm $18^{\circ} 17^{\prime} 24.25^{\prime \prime N}, 9^{\circ} 17^{\prime} 3.55^{\prime \prime O}, 996$ msnm $18^{\circ} 16^{\prime} 54.04 " \mathrm{~N}, 9^{\circ} 17^{\prime} 46.56^{\prime \prime O}, 985 \mathrm{msnm}$ $18^{\circ} 16^{\prime} 34.02^{\prime \prime N}, 99^{\circ} 14^{\prime} 45.59^{\prime \prime} \mathrm{O}, 1312 \mathrm{msnm}$

\section{Iguala de la Independencia}

$18^{\circ} 4^{\prime} 23.70 " \mathrm{~N}, 9^{\circ} 38^{\prime} 20.80^{\prime \prime} \mathrm{O}, 886 \mathrm{msnm}$

\section{Juan R. Escudero}

$17^{\circ} 10^{\prime} 47.03^{\prime \prime N}, 99^{\circ} 32^{\prime} 43.33^{\prime \prime O}, 342 \mathrm{msnm}$ $17^{\circ} 9^{\prime} 5.58^{\prime \prime N}, 9^{\circ} 34^{\prime} 6.53 " \mathrm{O}, 224 \mathrm{msnm}$ $17^{\circ} 9^{\prime} 4.86^{\prime \prime N}, 9^{\circ} 33^{\prime} 48.82^{\prime \prime O}, 175 \mathrm{msnm}$ $17^{\circ} 9^{\prime} 3.99^{\prime \prime N}, 99^{\circ} 33^{\prime} 48.88^{\prime \prime O}, 183 \mathrm{msnm}$ $17^{\circ} 9^{\prime} 1.58^{\prime \prime N}, 9^{\circ} 34^{\prime} 16.97 " \mathrm{O}, 178 \mathrm{msnm}$ $17^{\circ} 8^{\prime} 50.60^{\prime \prime} \mathrm{N}, 9^{\circ} 33^{\prime} 17.03^{\prime \prime O}, 202 \mathrm{msnm}$ $17^{\circ} 6^{\prime} 15.80^{\prime \prime N}, 99^{\circ} 32^{\prime} 14.93^{\prime \prime O}, 245 \mathrm{msnm}$ $17^{\circ} 5^{\prime} 35.74^{\prime \prime N}, 99^{\circ} 33^{\prime} 3.30^{\prime \prime O}, 120 \mathrm{msnm}$ $17^{\circ} 5^{\prime} 17.20^{\prime \prime N}, 9^{\circ} 32^{\prime} 35.34^{\prime \prime O}, 129 \mathrm{msnm}$ $17^{\circ} 4^{\prime} 37.30^{\prime \prime} \mathrm{N}, 9^{\circ} 29^{\prime} 37.43^{\prime \prime O}, 324 \mathrm{msnm}$ $17^{\circ} 4^{\prime} 33.19^{\prime \prime N}, 99^{\circ} 30^{\prime} 7.30^{\prime \prime O}, 201 \mathrm{msnm}$ $17^{\circ} 3^{\prime} 43.56^{\prime \prime N}, 9^{\circ} 29^{\prime} 59.68^{\prime \prime} \mathrm{O}, 167 \mathrm{msnm}$ $17^{\circ} 2^{\prime} 9.20^{\prime \prime N}, 99^{\circ} 32^{\prime} 51.68^{\prime \prime O}, 144 \mathrm{msnm}$

\section{La Unión de Isidoro Montes de Oca}

$18^{\circ} 1^{\prime} 55.97^{\prime \prime} \mathrm{N}, 101^{\circ} 45^{\prime} 5.05^{\prime \prime O}, 100 \mathrm{msnm}$ $18^{\circ} 00^{\prime} 57.45 " \mathrm{~N}, 102^{\circ} 0 ' 19.26^{\prime \prime} \mathrm{O}, 44 \mathrm{msnm}$ $18^{\circ} 0 ' 55.90^{\prime \prime} \mathrm{N}, 102^{\circ} 0^{\prime} 18.766^{\prime \prime} \mathrm{O}, 41 \mathrm{msnm}$ $17^{\circ} 56^{\prime} 47.56^{\prime \prime} \mathrm{N}, 101^{\circ} 50^{\prime} 42.67^{\prime \prime O}, 45 \mathrm{msnm}$ $17^{\circ} 56^{\prime} 0.87^{\prime \prime} \mathrm{N}, 101^{\circ} 48^{\prime} 43.01^{\prime \prime} \mathrm{O}, 57 \mathrm{msnm}$ $17^{\circ} 55^{\prime} 33.30^{\prime \prime} \mathrm{N}, 101^{\circ} 46^{\prime} 31.45^{\prime \prime O}, 76 \mathrm{msnm}$ $17^{\circ} 51^{\prime} 42.79^{\prime \prime} \mathrm{N}, 101^{\circ} 37^{\prime} 12.45^{\prime \prime O}, 299 \mathrm{msnm}$

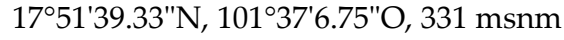
$17^{\circ} 51^{\prime 2} 2.69^{\prime \prime} \mathrm{N}, 101^{\circ} 37^{\prime} 0.11^{\prime \prime O}, 332 \mathrm{msnm}$ $17^{\circ} 47^{\prime} 44.31^{\prime \prime N}, 101^{\circ} 42^{\prime} 43.65^{\prime \prime O}, 64 \mathrm{msnm}$

\section{Malinaltepec}

$16^{\circ} 57^{\prime} 24.07^{\prime \prime} \mathrm{N}, 98^{\circ} 43^{\prime} 42.32^{\prime \prime} \mathrm{O}, 689 \mathrm{msnm}$

\section{Olinalá}

$\begin{array}{lll}12^{(2)} & 10 & 2017 \\ 8^{(1)} & 7 & 2017 \\ 2^{(1)} & 1 & 2017 \\ 10 & 10 & 2013\end{array}$

$9^{(1)}$ 8

2014

$\begin{array}{lll}32^{(2)} & 32 & 2017 \\ 35^{(2)} & 33 & 2016 \\ 73^{(2)} & 71 & 2016 \\ 34^{(2)} & 32 & 2017 \\ 77^{(1)} & 76 & 2017 \\ 57^{(1)} & 56 & 2017 \\ 17^{(2)} & 15 & 2013 \\ 4 & 4 & 2013 \\ 4 & 4 & 2014 \\ 13^{(2)} & 11 & 2014 \\ 0 & 0 & 2014 \\ 2 & 2 & 2014 \\ 22 & 22 & 2014\end{array}$

$\begin{array}{lll}47^{(4)} & 43 & 2014 \\ 4 & 4 & 2014 \\ 0 & 0 & 2014 \\ 5 & 5 & 2014 \\ 87^{(2)} & 85 & 2014 \\ 0 & 0 & 2014 \\ 2^{(1)} & 1 & 2017 \\ 5^{(1)} & 4 & 2017 \\ 9^{(2)} & 7 & 2017 \\ 38^{(2)} & 36 & 2014\end{array}$

3

3

2013
21

2 


\section{Ometepec}

$16^{\circ} 40^{\prime} 51.78^{\prime \prime} \mathrm{N}, 98^{\circ} 15^{\prime} 27.044^{\prime O}, 510 \mathrm{msnm}$ $16^{\circ} 39^{\prime} 48.11^{\prime \prime N}, 98^{\circ} 18^{\prime} 22.50^{\prime \prime} \mathrm{O}, 347 \mathrm{msnm}$ $16^{\circ} 39^{\prime} 21.82^{\prime \prime N}, 98^{\circ} 14^{\prime} 14.87^{\prime \prime} \mathrm{O}, 868 \mathrm{msnm}$ $16^{\circ} 38^{\prime} 16.07^{\prime \prime} \mathrm{N}, 98^{\circ} 17^{\prime} 8.47^{\prime \prime O}, 375 \mathrm{msnm}$ $16^{\circ} 37^{\prime} 8.38^{\prime \prime N}, 98^{\circ} 13^{\prime} 19.16^{\prime \prime O}, 375$ msnm $16^{\circ} 37^{\prime} 30.33^{\prime \prime N}, 98^{\circ} 13^{\prime} 27.06^{\prime \prime O}, 417$ msnm $16^{\circ} 37^{\prime} 19.56^{\prime \prime} \mathrm{N}, 98^{\circ} 13^{\prime} 28.61^{\prime \prime O}, 428 \mathrm{msnm}$ $16^{\circ} 37^{\prime} 30.07^{\prime \prime N}, 98^{\circ} 15^{\prime} 0.52^{\prime \prime O}, 387$ msnm $16^{\circ} 37^{\prime} 29.85^{\prime \prime} \mathrm{N}, 98^{\circ} 16^{\prime} 28.14^{\prime \prime} \mathrm{O}, 289 \mathrm{msnm}$ $16^{\circ} 37^{\prime} 4.61 " \mathrm{~N}, 98^{\circ} 15^{\prime} 47.73^{\prime \prime O}, 316 \mathrm{msnm}$ $16^{\circ} 37^{\prime} 3.80^{\prime \prime} \mathrm{N}, 98^{\circ} 24^{\prime} 3.24^{\prime \prime O}, 42 \mathrm{msnm}$ $16^{\circ} 36^{\prime} 54.00^{\prime \prime} \mathrm{N}, 98^{\circ} 13^{\prime} 16.89^{\prime \prime} \mathrm{O}, 378 \mathrm{msnm}$ $16^{\circ} 36^{\prime} 27.73^{\prime \prime N}, 98^{\circ} 14^{\prime} 14.89^{\prime \prime O}, 335 \mathrm{msnm}$ $16^{\circ} 36^{\prime} 5.94 " \mathrm{~N}, 98^{\circ} 13^{\prime} 48.52^{\prime \prime O}, 355 \mathrm{msnm}$ $16^{\circ} 35^{\prime} 56.14^{\prime \prime} \mathrm{N}, 98^{\circ} 12^{\prime} 5.13^{\prime \prime O}, 296$ msnm $16^{\circ} 35^{\prime} 51.30^{\prime \prime} \mathrm{N}, 98^{\circ} 15^{\prime} 47.91^{\prime \prime} \mathrm{O}, 297 \mathrm{msnm}$ $16^{\circ} 35^{\prime} 41.62^{\prime \prime} \mathrm{N}, 98^{\circ} 24^{\prime} 23.47^{\prime \prime} \mathrm{O}, 35 \mathrm{msnm}$ $16^{\circ} 34^{\prime} 57.44^{\prime \prime N}, 98^{\circ} 13^{\prime} 27.06^{\prime \prime O}, 287 \mathrm{msnm}$ $16^{\circ} 33^{\prime} 50.26^{\prime \prime} \mathrm{N}, 98^{\circ} 17^{\prime} 7.58^{\prime \prime} \mathrm{O}, 90 \mathrm{msnm}$

11

\section{Petatlán}

\section{San Luis Acatlán}

$16^{\circ} 49^{\prime} 38.27^{\prime \prime} \mathrm{N}, 98^{\circ} 38^{\prime} 24.22 " \mathrm{O}, 736 \mathrm{msnm}$ $16^{\circ} 49^{\prime} 10.52^{\prime \prime} \mathrm{N}, 98^{\circ} 40^{\prime} 29.93^{\prime \prime} \mathrm{O}, 638 \mathrm{msnm}$ $16^{\circ} 49^{\prime} 7.61 " \mathrm{~N}, 98^{\circ} 40^{\prime} 37.88^{\prime \prime O}, 585 \mathrm{msnm}$ $16^{\circ} 48^{\prime} 57.42^{\prime \prime} \mathrm{N}, 98^{\circ} 41^{\prime} 56.87^{\prime \prime} \mathrm{O}, 589 \mathrm{msnm}$ $16^{\circ} 48^{\prime} 54.07^{\prime \prime} \mathrm{N}, 98^{\circ} 40^{\prime} 45.98^{\prime \prime} \mathrm{O}, 648 \mathrm{msnm}$ $16^{\circ} 48^{\prime} 50.33^{\prime \prime} \mathrm{N}, 98^{\circ} 40^{\prime} 45.62^{\prime \prime} \mathrm{O}, 643 \mathrm{msnm}$ $16^{\circ} 48^{\prime} 11.46^{\prime \prime} \mathrm{N}, 98^{\circ} 41^{\prime} 10.83^{\prime \prime O}, 655 \mathrm{msnm}$ $16^{\circ} 47^{\prime} 22.85^{\prime \prime} \mathrm{N}, 98^{\circ} 41^{\prime} 47.33^{\prime \prime O}, 437 \mathrm{msnm}$ $16^{\circ} 46^{\prime} 53.83^{\prime \prime} \mathrm{N}, 98^{\circ} 44^{\prime} 35.544^{\prime \prime} \mathrm{O}, 260 \mathrm{msnm}$ $16^{\circ} 46^{\prime} 47.17^{\prime \prime} \mathrm{N}, 98^{\circ} 46^{\prime} 8.90^{\prime \prime} \mathrm{O}, 248 \mathrm{msnm}$ $16^{\circ} 46^{\prime} 37.19^{\prime \prime} \mathrm{N}, 98^{\circ} 44^{\prime} 56.77^{\prime \prime} \mathrm{O}, 283 \mathrm{msnm}$ $16^{\circ} 46^{\prime} 30.64^{\prime \prime N}, 98^{\circ} 46^{\prime} 5.36$ "O, 269 msnm

\section{San Marcos}

$17^{\circ} 1^{\prime} 48.40^{\prime \prime} \mathrm{N}, 99^{\circ} 30^{\prime} 21.29^{\prime \prime} \mathrm{O}, 182 \mathrm{msnm}$ $17^{\circ} 0^{\prime} 17.50 " \mathrm{~N}, 9^{\circ} 30^{\prime} 50.766^{\prime \prime} \mathrm{O}, 165 \mathrm{msnm}$ $16^{\circ} 59^{\prime} 7.01^{\prime \prime N}, 9^{\circ} 33^{\prime} 47.74 " \mathrm{O}, 328 \mathrm{msnm}$ $16^{\circ} 58^{\prime} 35.65^{\prime \prime N}, 99^{\circ} 32^{\prime} 55.72$ "O, 328 msnm $16^{\circ} 54^{\prime} 14.00^{\prime \prime} \mathrm{N}, 99^{\circ} 30^{\prime} 48.13$ "O, $213 \mathrm{msnm}$ $16^{\circ} 54^{\prime} 12.02^{\prime \prime} \mathrm{N}, 99^{\circ} 30^{\prime} 33.98^{\prime \prime} \mathrm{O}, 212 \mathrm{msnm}$ $16^{\circ} 54^{\prime} 10.77^{\prime \prime} \mathrm{N}, 9^{\circ} 30^{\prime} 10.26^{\prime \prime O}, 206 \mathrm{msnm}$ $16^{\circ} 53^{\prime} 55.18^{\prime \prime} \mathrm{N}, 9^{\circ} 31^{\prime} 4.70^{\prime \prime O}, 191 \mathrm{msnm}$ $16^{\circ} 54^{\prime} 3.38^{\prime \prime N}, 99^{\circ} 30^{\prime} 52.53 " \mathrm{O}, 194 \mathrm{msnm}$ $16^{\circ} 46^{\prime} 43.56^{\prime \prime} \mathrm{N}, 99^{\circ} 15^{\prime} 12.25^{\prime \prime} \mathrm{O}, 63 \mathrm{msnm}$ $16^{\circ} 46^{\prime} 30.65^{\prime \prime N}, 99^{\circ} 16^{\prime} 21.61^{\prime \prime O}, 118 \mathrm{msnm}$ $16^{\circ} 45^{\prime} 5.26^{\prime \prime} \mathrm{N}, 9^{\circ} 15^{\prime} 57.533^{\prime \prime O}, 42 \mathrm{msnm}$ $16^{\circ} 45^{\prime} 3.89^{\prime \prime} \mathrm{N}, 99^{\circ} 15^{\prime} 57.49^{\prime \prime} \mathrm{O}, 46 \mathrm{msnm}^{* *}$ $16^{\circ} 44^{\prime} 51.86^{\prime \prime} \mathrm{N}, 99^{\circ} 18^{\prime} 24.70^{\prime \prime} \mathrm{O}, 55 \mathrm{msnm}$ $16^{\circ} 44^{\prime} 51.44^{\prime \prime N}, 99^{\circ} 18^{\prime} 22.56^{\prime \prime O}, 43 \mathrm{msnm}$ $16^{\circ} 44^{\prime} 7.76^{\prime \prime} \mathrm{N}, 99^{\circ} 17^{\prime} 31.23^{\prime \prime O}, 34 \mathrm{msnm}$

$\begin{array}{lll}0 & 0 & 2017 \\ 3^{(1)} & 2 & 2017 \\ 12^{(1)} & 11 & 2017 \\ 35^{(1)} & 34 & 2017 \\ 7^{(1)} & 6 & 2017 \\ 5 & 5 & 2017 \\ 25 & 25 & 2017 \\ 56^{(1)} & 55 & 2017 \\ 21 & 21 & 2016 \\ 5^{(1)} & 4 & 2016 \\ 2^{(1)} & 2 & 2016 \\ 16^{(1)} & 15 & 2016\end{array}$

2017

2017

2017

2017

2017

2017

2016

2016

2016

2014 2014 2017 2017 2013 2013 2013 2013 2013 2015 2016 2016 2015 2016 2015 2015 
$16^{\circ} 44^{\prime} 6.42^{\prime \prime} \mathrm{N}, 99^{\circ} 17^{\prime} 15.90^{\prime \prime} \mathrm{O}, 32 \mathrm{msnm}$ * $16^{\circ} 44^{\prime} 3.13^{\prime \prime N}, 9^{\circ} 17^{\prime} 23.77^{\prime \prime} \mathrm{O}, 32 \mathrm{msnm}$ $16^{\circ} 39^{\prime} 48.29^{\prime \prime} \mathrm{N}, 9^{\circ} 14^{\prime} 35.35^{\prime \prime} \mathrm{O}, 12 \mathrm{msnm}$ $16^{\circ} 39^{\prime} 28.65^{\prime \prime} \mathrm{N}, 99^{\circ} 13^{\prime} 8.19^{\prime \prime} \mathrm{O}, 10 \mathrm{msnm}$

\section{Taxco de Alarcón}

$18^{\circ} 40^{\prime} 0.26^{\prime \prime N}, 9^{\circ} 29^{\prime} 20.63 " \mathrm{O}, 1150 \mathrm{msnm}$ $18^{\circ} 40^{\prime} 0.26^{\prime \prime N}, 99^{\circ} 28^{\prime} 44.65^{\prime \prime O}, 1253 \mathrm{msnm}$ $18^{\circ} 37^{\prime} 0.19^{\prime \prime} \mathrm{N}, 99^{\circ} 32^{\prime} 15.68^{\prime \prime} \mathrm{O}, 1444 \mathrm{msnm}$ $18^{\circ} 34^{\prime} 5.36^{\prime \prime N}, 9^{\circ} 33^{\prime} 36.00^{\prime \prime O}, 1551 \mathrm{msnm}$ 18³3'3.22"N, 99॰32'31.00"O, 1598 msnm $18^{\circ} 32^{\prime} 54.66^{\prime \prime} \mathrm{N}, 9^{\circ} 33^{\prime} 37.59^{\prime \prime} \mathrm{O}, 1495 \mathrm{msnm}$ $18^{\circ} 32^{\prime} 50.47^{\prime \prime} \mathrm{N}, 9^{\circ} 33^{\prime} 49.57^{\prime \prime O}, 1449 \mathrm{msnm}$ $18^{\circ} 32^{\prime} 6.36 " \mathrm{~N}, 9^{\circ} 36^{\prime} 11.77^{\prime \prime O}, 1516 \mathrm{msnm}$

\section{Tecoanapa}

$16^{\circ} 58^{\prime} 3.14 " \mathrm{~N}, 99^{\circ} 14^{\prime} 27.79$ "O, $^{\circ} 357 \mathrm{msnm}$ $16^{\circ} 56^{\prime} 25.16^{\prime \prime} \mathrm{N}, 99^{\circ} 15^{\prime} 8.41$ "O, $550 \mathrm{msnm}$

\section{Zihuatanejo de Azueta}

$17^{\circ} 46^{\prime} 8.72^{\prime \prime} \mathrm{N}, 101^{\circ} 31^{\prime} 1.999^{\prime O}, 164 \mathrm{msnm}$ $17^{\circ} 45^{\prime} 47.31^{\prime \prime} \mathrm{N}, 101^{\circ} 26^{\prime} 58.00^{\prime \prime} \mathrm{O}, 563 \mathrm{msnm}$ $17^{\circ} 45^{\prime} 46.80^{\prime \prime} \mathrm{N}, 101^{\circ} 26^{\prime} 58.45^{\prime \prime} \mathrm{O}, 534 \mathrm{msnm}$ $17^{\circ} 44^{\prime} 55.93^{\prime \prime} \mathrm{N}, 101^{\circ} 26^{\prime} 10.00 " \mathrm{O}, 500 \mathrm{msnm}$ $17^{\circ} 44^{\prime} 30.16^{\prime \prime N}, 101^{\circ} 28^{\prime} 8.04 " \mathrm{O}, 507$ msnm
$18^{(5) *}$

$4^{(1)}$

32

2

13

3

32

2

2015

2016

2017

2016

2014

2014

2014

2014

2017

2016

2016

2014

$12^{(1)}$

3

11

2017

2017

$\begin{array}{lll}64^{(3)} & 61 & 2016 \\ 22^{(1)} & 21 & 2016 \\ 5 & 5 & \\ 49^{(2)} & 47 & 2016 \\ 1^{(1)} & 0 & 2016\end{array}$

El número entre “(1...)" corresponde al número de especímenes enviados a laboratorio.

El signo "*" corresponde al número de casos positivos. 